\title{
Cellular response to high pulse repetition rate nanosecond pulses varies with fluorescent marker identity
}

\author{
Zachary A. Steelman, ${ }^{\text {a* }}$ Gleb P. Tolstykh, ${ }^{b}$ Hope T. Beier, ${ }^{c}$ and Bennett L. Ibey \\ ${ }^{a}$ Duke University, Department of Biomedical Engineering, 101 Science Drive, Durham, NC, USA, \\ ${ }^{\mathrm{b}}$ General Dynamics Information Technology, Fort Sam Houston, TX, USA \\ ${ }^{\mathrm{c}}$ Radio Frequency Bioeffects Branch, Air Force Research Laboratories, Ft. Sam Houston, San Antonio, TX
}

\begin{abstract}
Nanosecond electric pulses (nsEP's) are a well-studied phenomena in biophysics that cause substantial alterations to cellular membrane dynamics, internal biochemistry, and cytoskeletal structure, and induce apoptotic and necrotic cell death. While several studies have attempted to measure the effects of multiple nanosecond pulses, the effect of pulse repetition rate (PRR) has received little attention, especially at frequencies greater than $100 \mathrm{~Hz}$. In this study, uptake of Propidium Iodide, FM 1-43, and YO-PRO-1 fluorescent dyes in CHO-K1 cells was monitored across a wide range of PRRs $(5 \mathrm{~Hz}$ to $500 \mathrm{KHz})$ using a laser-scanning confocal microscope in order to better understand how high frequency repetition rates impact induced biophysical changes. We show that frequency trends depend on the identity of the dye under study, which could implicate transmembrane protein channels in the uptake response due to their chemical selectivity. Finally, YO-PRO-1 fluorescence was monitored in the presence of Gadolinium $\left(\mathrm{Gd}^{3+}\right)$, Ruthenium Red, and in calcium-free solution to elucidate a mechanism for its unique frequency trend.
\end{abstract}

Keywords: Nanosecond pulse, nsEP, CHO-K1, fluorescence microscopy, confocal microscopy

Abbreviations: PRR Pulse repetition rate; nsEP Nanosecond electric pulse

*Corresponding Author E-mail: zachary.steelman@duke.edu , Telephone: 713-299-3143

\section{Introduction}

Electroporation is an established biophysical technique in which cells are subjected to a highvoltage electric field, inducing myriad cytoskeletal, chemical, and receptor-mediated effects. Nanoporation, a more recent field of interest, expands the concept of electroporation to pulses which last on the order of tens to hundreds of nanoseconds. Once thought to be simply an extension of electroporation, nanoporation has been shown to exhibit substantially different phenomena from classical electroporation experiments [1-5]. Common biological responses to nanosecond electrical pulses include nanopore formation, phospholipid scrambling, cell blebbing 
and swelling, activation of the $\mathrm{IP}_{3} / \mathrm{DAG}$ pathway, bursts of intracellular calcium, and apoptotic or necrotic cell death [6-10].

Varying the electric field dosage through the use of multiple pulses is a common technique in the study of nanoporation bioeffects $[11,12]$. However, significant variation exists in the literature as to the pulse repetition rate (PRR) used in various studies (here, PRR refers to the reciprocal time between the start of two consecutive, square nsEP waveforms). While some studies have begun to understand the role that PRR plays in the biophysical effects of nanoporation, early experiments have been limited to a narrow $(1-30 \mathrm{~Hz})$ range of frequencies [13]. To study the wider effects of PRR, we applied 10 consecutive pulses (200V, $600 \mathrm{~ns}$ each) to CHO-K1 cells, with the PRR swept between $5 \mathrm{~Hz}$ and $500 \mathrm{KHz}$. The percentage change in fluorescence $(\Delta \mathrm{F} / \mathrm{F})$ was measured for all cells within the field.

Conventionally, Propidium Iodide and YO-PRO-1 have been the most commonly used fluorophores in the study of nanoporation bioeffects. This has principally been due to their chemical similarity, with a difference in molecular size (PI is larger) allowing for exclusionary sizing of nanopores. In this study, we use these two markers, along with the smaller molecule FM 1-43, to give a range of molecular sizes in which to study membrane permeabilization. All experiments were repeated using one of these dyes to probe the cellular response. To further study the phenomenon, YO-PRO-1 was used to study the effects of pharmacologic agents (Gadolinium, Ruthenium Red) and ion conditions $\left(\mathrm{Ca}^{2+}\right.$-free media) on the frequency response. We hypothesized that fluorophore uptake would follow the trends observed at low frequencies, with a reduction in uptake at high frequencies. We also speculated that the response of cells to very high PRR $(500 \mathrm{KHz})$ would approach that of a continuous pulse of equivalent energy, as the timescale of delivery approaches that of a single long pulse. 


\section{Materials and Methods}

\section{Cell Culture}

CHO-K1 cells transfected with a human muscarinic type 1 (hM1) receptor were cultured using standard protocols. The cells were transfected to provide continuity with previous experiments, as well as to allow for drug treatment with agonists of the $\mathrm{hM}_{1}$ receptor in further studies. The culture used a complete growth medium consisting of Ham's F-12K media supplemented with $10 \%$ fetal bovine serum, $1 \%$ penicillin/streptomycin antibiotic, and $0.48 \%$ G418 antibiotic (Life Technologies) for transfection stability. Cells were plated on $35 \mathrm{~mm}$ glass-bottomed dishes (MatTek No. 0, Ashland, MA) coated with poly-D-lysine approximately 18 hours prior to staining and exposure, and left in complete growth media in an incubator overnight.

\section{Fluorescence Imaging}

Immediately before imaging, media was removed, and cells were washed with $2 \mathrm{~mL}$ calciumcontaining standard outside solution consisting of $2 \mathrm{mM} \mathrm{MgCl}_{2}, 5 \mathrm{mM} \mathrm{KCl}, 10 \mathrm{mM} \mathrm{HEPES}$, $10 \mathrm{mM}$ glucose, $2 \mathrm{mM} \mathrm{CaCl}_{2}$, and $0.135 \mathrm{M} \mathrm{NaCl}$ in deionized water. The dish was then filled with $3 \mathrm{~mL}$ of standard outside solution with one of three molecular probes $(2 \mu \mathrm{M}$ YO-PRO- $1,3 \mu \mathrm{M}$ Propidium Iodide, or $8 \mu \mathrm{M}$ FM 1-43, Life Technologies). All exposures were completed within 45 minutes of media removal.

For all exposures containing $\mathrm{Gd}^{3+}$, the standard outside solution was supplemented with $300 \mu \mathrm{M}$ $\mathrm{Gd}^{3+}$ in both the washing and imaging steps. In Ruthenium Red experiments, the Gadolinium protocol was repeated, substituting $50 \mu \mathrm{M}$ Ruthenium Red for $\mathrm{Gd}^{3+}$. For calcium-free exposures, the standard outside solution was prepared as described above, with $2 \mathrm{mM} \mathrm{CaCl}_{2}$ replaced with 
$2 \mathrm{mM}$ sodium EGTA. In these cases, $2 \mu \mathrm{M}$ YO-PRO-1 was added to the imaging solution as before.

\section{Pulsing System}

Electric pulses were delivered to the cells from a Velonix high power pulse generator (Velonix, Model 360) via a custom tungsten wire electrode pair. The electrodes were made using 0.005 inch diameter parallel tungsten wires insulated with 0.0075 inch diameter polyimide tubing. The tips of the probe were left uninsulated, and submerged in the outside solution as previously described [14]. A Sutter MPC-200 micromanipulator (Sutter Instrument Co., Novato, CA) was used to position the electrode pair $50 \mu \mathrm{m}$ above the surface of the glass dish to facilitate uniform delivery of the field, and the output of the pulser was monitored with an oscilloscope to ensure accurate pulse delivery. The probe separation was $110 \pm 5 \mu \mathrm{m}$, and FDTD modeling [12] confirms that this equates to an electric field strength at the location of the cells of $3.1 \mathrm{kV} / \mathrm{cm}$. A Stanford Delay Generator (Stanford Research Systems, Model DG535) was used to coordinate timing between the microscope and the pulsing system. At $\mathrm{t}=0$ seconds, the Delay Generator triggered the laser scanning confocal microscope (Carl Zeiss LSM 710, Oberkochen, Germany) to begin two-channel acquisition of Differential Interference Contrast (DIC) and laser scanning fluorescence images at a rate of 1 image/second. An excitation wavelength of $488 \mathrm{~nm}$ was used for all 3 dyes, with emission filters chosen by Zeiss software to optimize the collected signal. Five seconds later, a trigger signal activated an HP pulse generator (Model HP 8112A, $50 \mathrm{MHz}$ ) to control the PRR and number of pulses. This signal was then sent to the external trigger channel of the pulse generator, which delivered 200V pulses at the set PRR. The output of the Velonix was monitored on a $500 \mathrm{MHz}$ Tektronix oscilloscope (Tektronix, Model TDS 3054B) using a 100X voltage dividing probe. 


\section{Exposure Conditions and Data Analysis}

In order to effectively study pulse repetition frequency, PRRs were chosen across several orders of magnitude. Prior to the experiment, it was determined that a PRR of $500 \mathrm{KHz}$ was the fastest permissible timescale before noticeable deformation of the pulse waveform was observed. For this reason, $500 \mathrm{KHz}$ was selected as the fastest PRR for study. Ten 200 Volt pulses applied at PRRs of $5 \mathrm{~Hz}, 500 \mathrm{~Hz}, 50 \mathrm{KHz}, 250 \mathrm{KHz}$, and $500 \mathrm{KHz}$ were examined. Our initial hypothesis was that ten fast exposures $(500 \mathrm{KHz}, 600 \mathrm{~ns}$ each) would approximate a single longer pulse (6 $\mu$ s duration) of equivalent energy. To test this, a single $6 \mu$ s pulse was also studied. Multiple plates were prepared, and each of the seven exposure conditions (five PRRs, one $6 \mu$ s pulse, and one sham) were randomized and applied on each plate until 20-40 cells were imaged per condition. All exposures were taken within 45 minutes of media removal, and repeated for all three fluorescent dyes. In the case of $\mathrm{Gd}^{3+}$, Ruthenium Red, and calcium-free conditions, the seven exposure conditions were randomly split across two plates, and each plate was imaged within 15 minutes of washing to minimize cell alteration or death due to prolonged exposure to these conditions.

For each exposure, phase contrast T-PMT and laser scanning fluorescence images were taken for 5 seconds prior to and 30 seconds after the initiation of the pulse train, at a rate of 1 combined image/second. Cell borders were selected from the DIC image using Image $(\mathrm{NIH})$ and mean intracellular fluorescence was calculated from the fluorescence channel for each cell at every time point. Between 20 and 40 cells (typically 35) were analyzed for each exposure condition, and sham (unpulsed) cells were measured as a negative control. 


\section{Results}
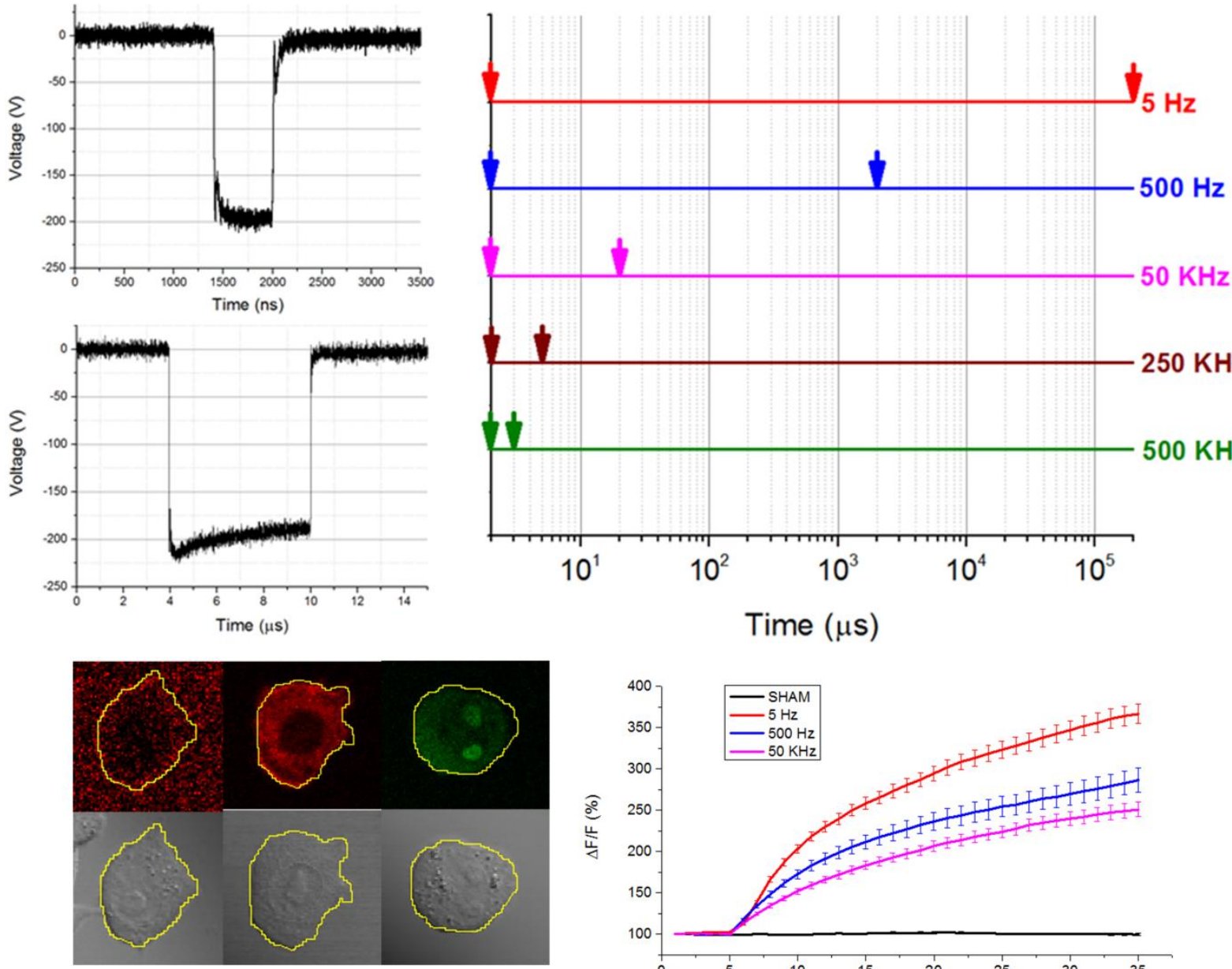

PI

FM 1-43 YO-PRO-1

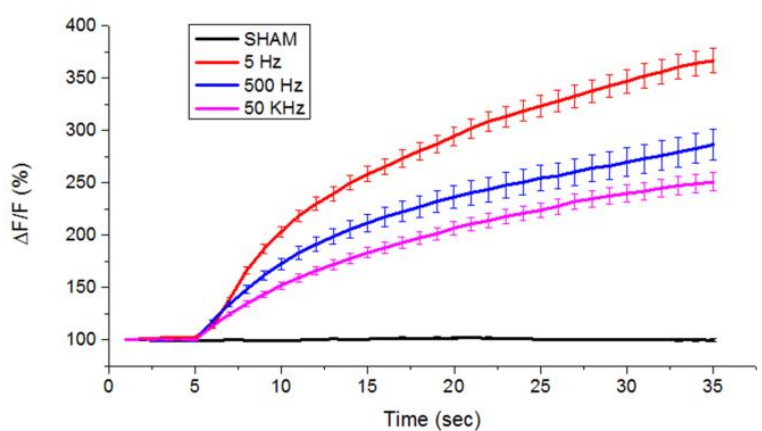

Fig. 1 (Top left) Representative 600 ns and $6 \mu$ s waveforms. (Top Right) Diagram showing various PRRs used with $600 \mathrm{~ns}$ pulses. (Bottom Left) Representative fluorescence and DIC images using each fluorophore. DIC images were used to guide selection of the cell border, and total internal fluorescence was measured within this border each second. (Bottom Right) Typical fluorescent response over time (YO-PRO-1 shown). Error bars indicate standard error of the mean.

Propidium Iodide uptake does not exhibit any strong frequency dependence.

Upon analysis of the data, cells bathed in a solution containing Propidium Iodide did not exhibit any strong dependence of dye uptake on pulse frequency in general. Plotted on the first row of

Fig. 2, little deviation occurred between fluorescence measurements for $5 \mathrm{~Hz}$ up to $500 \mathrm{KHz}$ 
application of ten 600 ns pulses. Significantly greater fluorescence was observed after application of one $6 \mu$ s pulse, which agrees with literature observations of increased cellular insult following microsecond pulsing [8]. Accordingly, a high PRR of $500 \mathrm{KHz}$ did not approximate a long pulse of equivalent energy $(\mathrm{P}<0.0001)$. While we initially hypothesized that a single continuous pulse would approximate a very fast PRR in associated bioeffects, in the case of PI, our hypothesis was proven incorrect.

FM 1-43 uptake decreases with faster PRR

In general, FM 1-43 uptake decreased monotonically with PRR (Fig. 2, second row). As with PI, a PRR of $500 \mathrm{KHz}$ did not recreate the effects of a single long pulse $(\mathrm{P}<0.0001)$. A single long pulse actually appears to approximate the effects of slower pulsing, with the response of cells to a single $6 \mu$ s pulse falling within the standard error of ten $600 \mathrm{~ns}$ pulses applied at $5 \mathrm{~Hz}$. The observation of decreasing membrane disruption at higher PRRs is in agreement with recent results [13] in which fluorophore uptake diminished with PRR, albeit at sub-50 Hz pulse rates. 

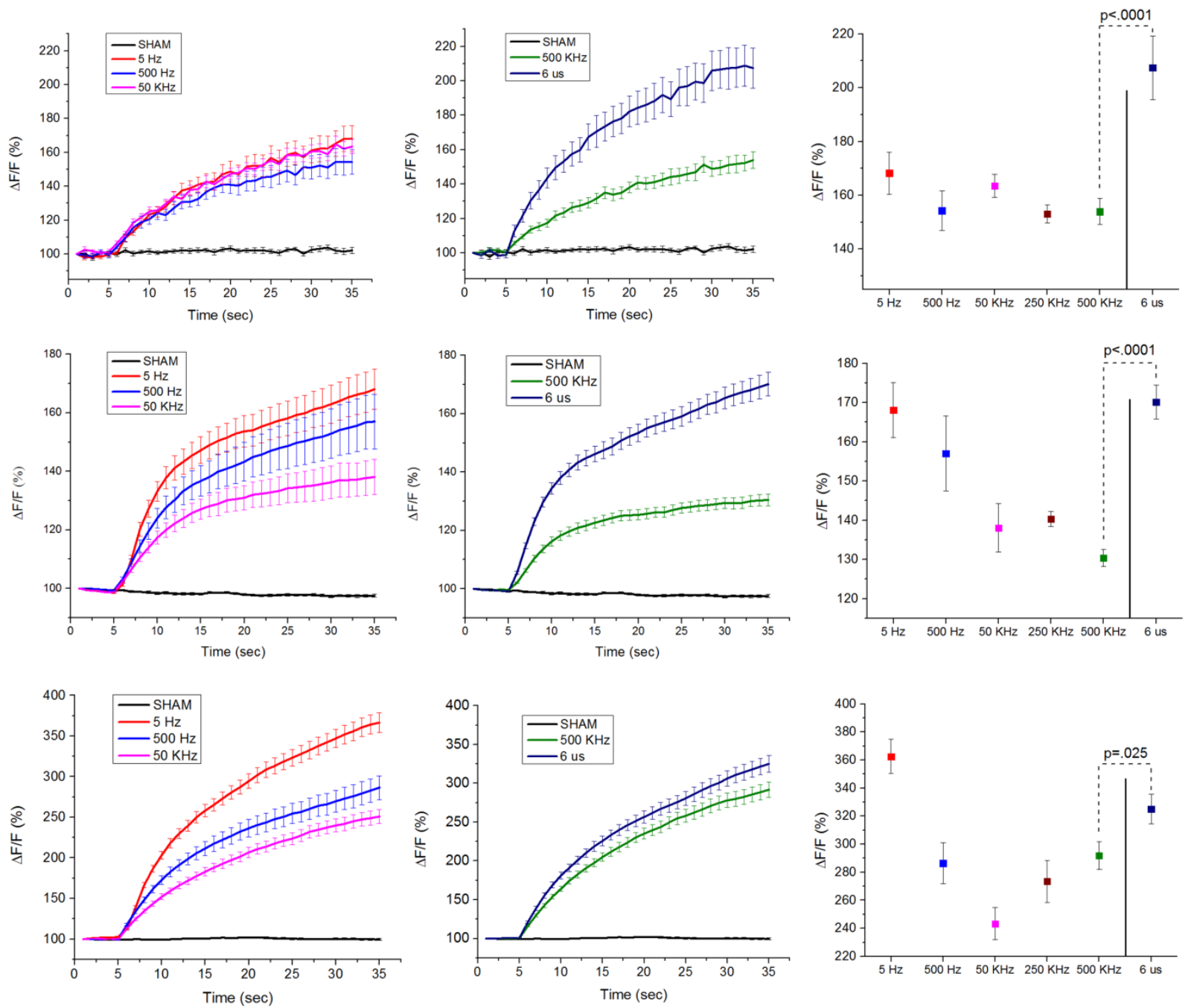

Fig. 2 (Top row) Fluorescence response of PI after ten 600 ns pulses and one $6 \mu$ s pulse. Ten pulses delivered at $500 \mathrm{KHz}$ do not approximate a single $6 \mu$ s pulse, $\mathrm{P}<0.0001$. For PI, no frequency dependence is observed. (Center Row) Fluorescence response of FM 1-43 under the same conditions. A PRR of $500 \mathrm{KHz}$ still does not approximate a $6 \mu$ s pulse, $\mathrm{P}<0.0001$. An inverse relationship is observed between frequency and FM 1-43 uptake. (Bottom Row) Fluorescence response of YO-PRO-1. Ten $600 \mathrm{~ns}$ pulses delivered at $500 \mathrm{KHz}$ and a single $6 \mu$ s pulse appear similar, and are only barely statistically significant $(\mathrm{P}=0.025)$ despite large $\mathrm{N}(\sim 36)$. The relationship between uptake and PRR appears to reverse near $50 \mathrm{KHz}$.

YO-PRO-1 uptake exhibits a trend reversal after $50 \mathrm{KHz}$

YO-PRO-1 exhibits a unique frequency response when compared to the first two dyes (Fig. 2,

row three). At low PRRs, YO-PRO-1 uptake decreases with pulse frequency, similar to the response of cells exposed to FM 1-43. Above $50 \mathrm{KHz}$, however, this trend reverses itself, with a 
monotonic increase in fluorescence up to $500 \mathrm{KHz}$. A $500 \mathrm{KHz}$ pulse application much more closely approximates the effects of a single long pulse ( $\mathrm{P}=0.025, \mathrm{~N}=36$ and 37 respectively), although slower pulses of 5-500 Hz also approximate a longer pulse. This two-part response is particularly interesting, as it could reflect the presence of two "regimes" in which high and low frequency pulse applications elicit similar biological responses in exposed cells.

$G^{3+}$ subdues most YO-PRO-1 uptake across frequencies

Cells exposed to $300 \mu \mathrm{M} \mathrm{Gd}{ }^{3+}$ exhibited drastically reduced fluorescence across all exposures. This result is in agreement with recently published literature, in which Gadolinium was shown to reduce the post-pulse permeability of the cell to Propidium Iodide [15]. Final percent fluorescence values after 30 seconds were less than $150 \%$ for all frequency conditions in $\mathrm{Gd}^{3+}$, compared to $250-400 \%$ changes observed in standard imaging solution. Intriguingly, the "trend reversal" effect previously observed with YO-PRO-1 is still present in $\mathrm{Gd}^{3+}$-exposed cells, although the reversal occurs at $250 \mathrm{KHz}$ instead of $50 \mathrm{KHz}$. The effects of one $6 \mu$ s pulse in $\mathrm{Gd}^{3+}$-exposed cells most closely approximate slower $(5 \mathrm{~Hz})$ PRRs.
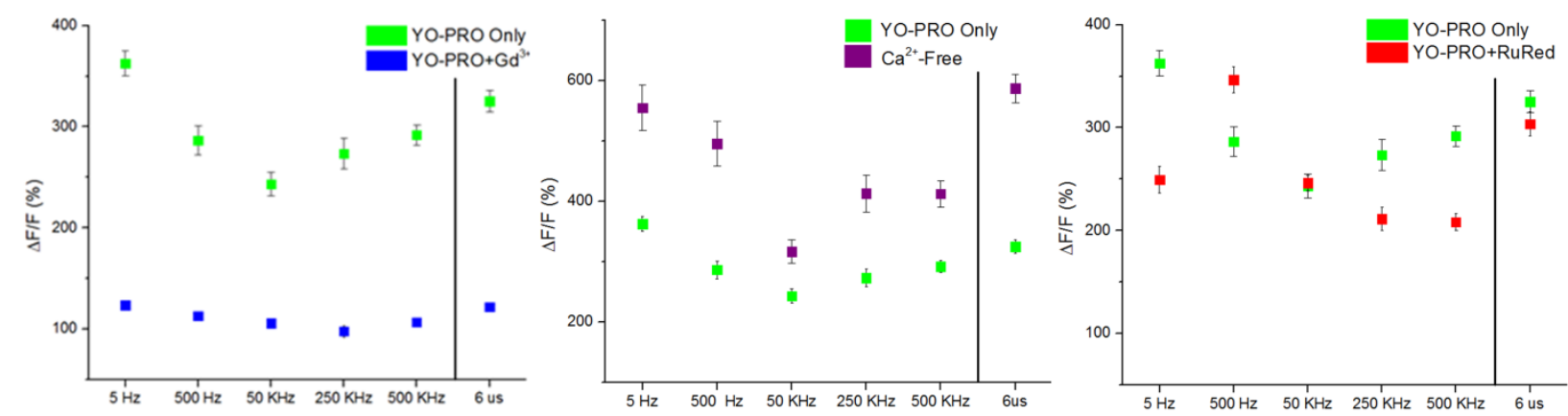

Fig. 3 (Left) YO-PRO-1fluorescence 30 seconds after pulsing at varying frequencies, with and without $300 \mu \mathrm{M}$ $\mathrm{Gd}^{3+}$. $\mathrm{Gd}^{3+}$ drastically reduces YO-PRO-1 uptake. (Middle) YO-PRO-1 fluorescence with and without calcium in the imaging buffer. A lack of calcium enhanced the nanoporation effect. (Right) The frequency response is modulated by Ruthenium Red, a nonspecific calcium channel blocker. 
Pulsing in $\mathrm{Ca}^{2+}$-free media increases YO-PRO-1 uptake

In the case of pulsing with zero calcium present in the imaging buffer, the percentage change in YO-PRO-1 fluorescence increased across all exposure conditions versus the control. The previously noted trend reversal at $50 \mathrm{KHz}$ was again present. In the absence of calcium, the separation between $500 \mathrm{KHz}$ exposures and a single $6 \mu$ s pulse was particularly apparent.

\section{Ruthenium Red modulates the frequency dependence of YO-PRO-1 uptake}

The YO-PRO-1 frequency experiments were again repeated, this time in the presence of Ruthenium Red, another nonspecific calcium channel blocker [16]. In most frequency regimes, a slight decrease in fluorescence was observed. However, one frequency $(50 \mathrm{KHz})$ showed negligible change, and another $(500 \mathrm{~Hz})$ actually showed an increase in fluorescence after 30 seconds. In a similar fashion to the calcium-free exposures, the bioeffects associated with 500 KHz PRR no longer approximate a continuous long pulse, despite this being the case using YOPRO-1 without any channel mediators.

\section{Discussion}

Because our analysis relied on manually selecting cell borders, there was an initial concern that cell swelling, often observed during nsPEF exposure, would contribute to a significant loss of signal as cells swelled outside of their initial location. While PI and YO-PRO-1 tend to fluoresce strongly at the nucleus, we were concerned with FM 1-43, due to the tendency of this molecule to embed partially in the plasma membrane. To quantify this effect, a random subset of 6-10 cells from every exposure condition in this study was retroactively analyzed to quantify cell swelling, and the results were tabulated in Figure 4. 

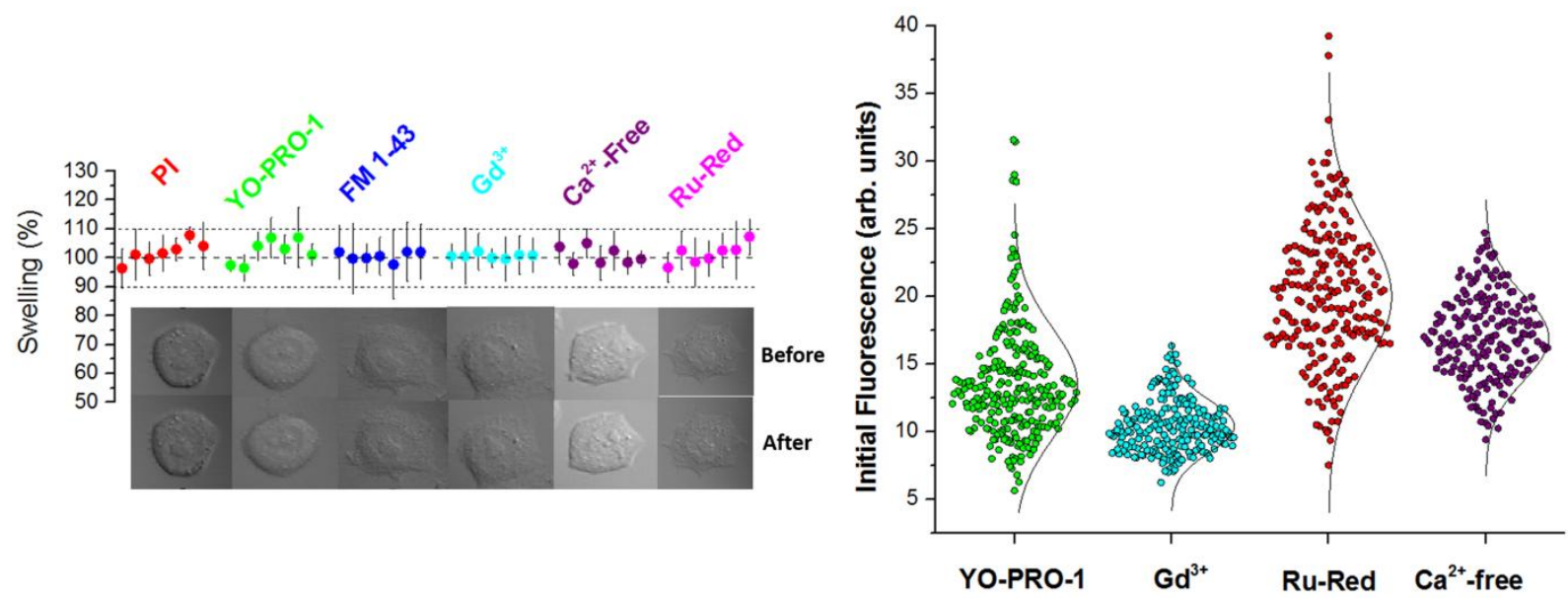

Fig. 4 (Left) Cell swelling, by post-pulse cell area over pre-pulse area. Mean swelling for all cases was $+1.28 \%$, with no condition eclipsing $+8 \%$. Typical DIC images of cells before and after pulsing are shown for $500 \mathrm{~Hz}$ exposures, with little morphological alteration. PRR within each of the six chemical conditions ("colors" above) are listed in their logical order left-to-right (Sham, $5 \mathrm{~Hz}, 500 \mathrm{~Hz}, 50 \mathrm{KHz}, 250 \mathrm{KHz}, 500 \mathrm{KHz}, 6 \mu \mathrm{s}$ ). (Right) Scatter plot of mean pre-pulse intracellular fluorescence of every cell in YO-PRO-1, fitted with a normal curve to show variance. Compared with standard YO-PRO-1 (13.5 \pm 4.2$)$, Gadolinium-modulated pre-pulse fluorescence was slightly reduced $(10.4 \pm 1.9)$ while Ruthenium Red $(20.0 \pm 5.0)$ and $\mathrm{Ca}^{2+}$-free media $(16.9 \pm 3.1)$ were increased slightly.

Cell area was quantified at time points five seconds before and 30 seconds after pulse application. Mean cell swelling was 1.28\% for all conditions, and less than $3 \%$ in 33 of 42 cases. For the fluorophore of greatest concern, FM 1-43, the mean swelling was the lowest, at $0.6 \%$ area change. Assuming a uniform fluorescence distribution (a generous assumption given the nuclear-centric signal) the mean error due to cell swelling in the worst case (YO-PRO-1) would be equivalent to its "lost" area of $2.3 \%$. Further, the large number of cells analyzed (20-40) can be reasonably expected to average away errors caused by variable swelling.

For certain conditions $\left(\mathrm{Gd}^{3+}\right.$, Ruthenium Red, $\mathrm{Ca}^{2+}$-free media) there was also a concern that the relative toxicity of the imaging environment would harm the cell, perturbing the integrity of the membrane and allowing YO-PRO-1 molecules to infiltrate the cell prior to exposure. In this case, a percentage change in fluorescence could misrepresent the number of fluorophores observed. In the interest of transparency, we investigated the pre-pulse intracellular fluorescence 
for the three exposure conditions of concern, as well as the standard YO-PRO-1 experiments as a control. Figure 4 (right) plots every cell's fluorescence before pulsing, with a normal curve fit to show the distribution. An examination of the data shows slight decreases in pre-pulse intensity associated with $\mathrm{Gd}^{3+}$ exposure, and slight increases associated with Ruthenium Red and $\mathrm{Ca}^{2+}$ free media. These could be associated with an over- and underestimation of the number of permeating molecules imaged, respectively. This analysis is intended chiefly to aid comparison between dissimilar exposure conditions, and has little bearing on the fluorescence responses within individual exposure conditions.

In general, we found that each fluorescent marker exhibited trends which were unique to the identity of the marker itself. Because the probes were chosen specifically for their distinct sizes (PI is approximately $1.5 \mathrm{~nm}$, YO-PRO-1 is approximately $1 \mathrm{~nm}$, and FM 1-43 is less than $1 \mathrm{~nm}$ in characteristic diameter $[17,18])$ it makes sense that physical effects associated with size, such as diffusion and nanopore permeability, may play a role in the uptake process. However, no obvious trend is occurring. PI, the largest molecule, shows essentially no frequency dependence, while FM 1-43, the smallest, shows a significant negative trend in uptake as PRR increases. YOPRO-1, of intermediate size, exhibited a trend reversal above $50 \mathrm{KHz}$. Overall, no clear trend is observed with relation to the molecular size of the probes.

One particularly captivating argument for the existence of unique frequency trends is the activity of protein channels, primarily for their potential to act as selective agents for the movement of specific fluorescent markers. It has been shown that $\mathrm{Gd}^{3+}$ has the ability to reduce the sensitivity of cells to bioeffects associated with nanoporation, including cell death [15]. However, $\mathrm{Gd}^{3+}$ is also a nonspecific cation channel blocker [19], usually of certain types of TRP and related channels. It has also been proven that YO-PRO-1 can enter the cell via non-selective, ATP- 
gated cation channels, such as the dilated form of the $\mathrm{P} 2 \mathrm{X}_{7}$ receptor $[20,21]$ or Pannexin 1 [22, 23]. We have demonstrated a substantial reduction in YO-PRO-1 uptake across the frequency spectrum using $300 \mu \mathrm{M} \mathrm{Gd}{ }^{3+}$, which agrees with reports from the literature suggesting an inhibition of nanoporation bioeffects. While several mechanisms are possible, the wellestablished role of $\mathrm{Gd}^{3+}$ as a channel blocker and its effectiveness at halting YO-PRO-1 uptake suggests that membrane channels could play a role in the uptake of YO-PRO-1 following nanosecond pulse exposure. This theory is further supported by the modulatory role of another channel blocker, Ruthenium Red, on the PRR spectrum, though more particular mechanisms must be investigated. Further studies in molecular dynamics, artificial membranes such as giant unilaminar vesicles, and the use of more specific channel blockers are necessary to determine the true mechanism of $\mathrm{Gd}^{3+}$ modulation of nanosecond pulse effects.

It is possible that the biochemistry of the three fluorophores used in this study contributed to their unique uptake characteristics in a manner separate from their size. However, YO-PRO-1, FM 1-43, and PI are all divalent cations in neutral solution and differ in molecular weight by less than $57 \mathrm{Da}$. Any difference in uptake caused by the fluorophore's biochemistry is likely specific, which could further support the involvement of channels.

Recent experiments have underscored the potential of mechanical stimuli to induce nanoporation-like effects [24]. The activation of stress-induced channels of the plasma membrane may provide a link between the two theories, and a new generation of mechanical spectroscopies with high spatial resolution could allow us to image these effects for the first time $[25,26]$.

To further understand the role of membrane proteins, we repeated the YO-PRO-1 exposures at all frequencies in calcium-free media. Since many membrane channels are calcium mediated, a 
modulated result would indicate that the presence of calcium plays a role in uptake, and thus, further implicate channels in at least a portion of the uptake mechanism. Interestingly, pulsing cells in calcium-free buffer actually increased YO-PRO-1 uptake throughout the frequency spectrum. Here, several mechanisms are possible. A lack of calcium on the membrane could alter the activity of channels which require calcium ions to operate. Lack of extracellular calcium could also lead to a depletion of intracellular calcium, inducing uptake pathways that favor calcium and thus YO-PRO-1 entry. Further, the absence of calcium may create a more favorable transport opportunity for YO-PRO-1 through passive calcium channels. Further work is needed to determine whether these mechanisms are feasible at a significant scale.

Finally, we note that while voltage-sensitive channels are not classically associated with CHOK1 cells, a review of the literature suggests that both TRP-like channels [27] and some voltage sensitive channels [28] are likely present in native CHO cells. The presence of these channels is interesting, as it provides a mechanism for $\mathrm{Gd}^{3+}$ inhibition of YO-PRO-1 entry which is grounded in known channel-mediated mechanisms.

To conclude, we have shown that PRR is an important parameter in the application of nanosecond pulsed electric fields, altering membrane disruption throughout the frequency spectrum. Further, we have proven that frequency trends in uptake are unique to each fluorophore, suggesting a molecular specificity to the mechanism underlying dye uptake. Finally, we have attempted to pinpoint the exact mechanism by exposing cells in $\mathrm{Gd}^{3+}$ and Ruthenium Red, as well as in the absence of calcium. We feel that this work is imperative to elucidate the fundamental biophysics of nanoporation, as well as to standardize the study of field-induced effects by showing the importance of an often ignored parameter. 


\section{Acknowledgments}

This research was supported in part by an appointment to the Student Research Participation Program at the U.S. Air Force Research Laboratory, 711th Human Performance Wing administered by the Oak Ridge Institute for Science and Education through an interagency agreement between the U.S. Department of Energy and USAFRL. This work was also supported by Air Force Office of Scientific Research LRIR 16RHCOR348.

\section{References}

[1] E. Tekle, H. Oubrahim, S.M. Dzekunov, J.F. Kolb, K.H. Schoenbach, P. Chock, Selective field effects on intracellular vacuoles and vesicle membranes with nanosecond electric pulses, Biophys. J., 89 (2005) 274-284.

[2] S.J. Beebe, J. White, P.F. Blackmore, Y. Deng, K. Somers, K.H. Schoenbach, Diverse effects of nanosecond pulsed electric fields on cells and tissues, DNA Cell Biol., 22 (2003) 785-796.

[3] S.J. Beebe, P.M. Fox, L.J. Rec, E.L.K. Willis, K.H. Schoenbach, Nanosecond, high-intensity pulsed electric fields induce apoptosis in human cells, FASEB J., 17 (2003) 1493-1495.

[4] P.T. Vernier, A. Li, L. Marcu, C.M. Craft, M.A. Gundersen, Ultrashort pulsed electric fields induce membrane phospholipid translocation and caspase activation: differential sensitivities of Jurkat T lymphoblasts and rat glioma C6 cells, IEEE Trans. Dielectr. Electr. Insul, 10 (2003) 795-809.

[5] Z.A. Steelman, G.P. Tolstykh, H.T. Beier, B.L. Ibey, High frequency application of nanosecond pulsed electric fields alters cellular membrane disruption and fluorescent dye uptake, SPIE BiOS, International Society for Optics and Photonics, 2016, pp. 97060W-97060W-97067.

[6] G.P. Tolstykh, H.T. Beier, C.C. Roth, G.L. Thompson, B.L. Ibey, 600ns pulse electric fieldinduced phosphatidylinositol 4, 5-bisphosphate depletion, Bioelectrochemistry, 100 (2014) 8087.

[7] H.T. Beier, C.C. Roth, G.P. Tolstykh, B.L. Ibey, Resolving the spatial kinetics of electric pulse-induced ion release, Biochem. Biophys. Res. Commun., 423 (2012) 863-866.

[8] P.T. Vernier, Y. Sun, L. Marcu, S. Salemi, C.M. Craft, M.A. Gundersen, Calcium bursts induced by nanosecond electric pulses, Biochem. Biophys. Res. Commun., 310 (2003) 286-295.

[9] P.T. Vernier, Y. Sun, L. Marcu, C.M. Craft, M.A. Gundersen, Nanosecond pulsed electric fields perturb membrane phospholipids in T lymphoblasts, FEBS Lett., 572 (2004) 103-108.

[10] Z.A. Steelman, G.P. Tolstykh, L.E. Estlack, C.C. Roth, B.L. Ibey, The role of PIP2 and the IP3/DAG pathway in intracellular calcium release and cell survival during nanosecond electric pulse exposures, SPIE BiOS, International Society for Optics and Photonics, 2015, pp. 932611932611-932616.

[11] B.L. Ibey, C.C. Roth, A.G. Pakhomov, J.A. Bernhard, G.J. Wilmink, O.N. Pakhomova, Dose-dependent thresholds of 10-ns electric pulse induced plasma membrane disruption and cytotoxicity in multiple cell lines, PLoS One, 6 (2011) e15642. 
[12] B.L. Ibey, D.G. Mixon, J.A. Payne, A. Bowman, K. Sickendick, G.J. Wilmink, W.P. Roach, A.G. Pakhomov, Plasma Membrane Permeabilization by Trains of Ultrashort Electric Pulses, Bioelectrochemistry (Amsterdam, Netherlands), 79 (2010) 114-121.

[13] P. Lamberti, S. Romeo, A. Sannino, L. Zeni, O. Zeni, The Role of Pulse Repetition Rate in nsPEF-induced Electroporation: A Biological and Numerical Investigation, IEEE Trans. Biomed. Eng., (2015).

[14] A.G. Pakhomov, A.M. Bowman, B.L. Ibey, F.M. Andre, O.N. Pakhomova, K.H. Schoenbach, Lipid nanopores can form a stable, ion channel-like conduction pathway in cell membrane, Biochem. Biophys. Res. Commun., 385 (2009) 181-186.

[15] F.M. André, M.A. Rassokhin, A.M. Bowman, A.G. Pakhomov, Gadolinium blocks membrane permeabilization induced by nanosecond electric pulses and reduces cell death, Bioelectrochemistry, 79 (2010) 95-100.

[16] C.L. Moore, Specific inhibition of mitochondrial $\mathrm{Ca}++$ transport by ruthenium red, Biochem. Biophys. Res. Commun., 42 (1971) 298-305.

[17] S.J. Beebe, Y.-J. Chen, N.M. Sain, K.H. Schoenbach, S. Xiao, Transient features in nanosecond pulsed electric fields differentially modulate mitochondria and viability, PLoS One, 7 (2012) e51349.

[18] A.M. Bowman, O.M. Nesin, O.N. Pakhomova, A.G. Pakhomov, Analysis of Plasma Membrane Integrity by Fluorescent Detection of Tl(+) Uptake, J. Membr. Biol., 236 (2010) 1526.

[19] J. Malysz, D. Richardsons, L. Farraway, J.D. Huizinga, M.-O. Christen, Generation of slow wave type action potentials in the mouse small intestine involves a non-L-type calcium channel, Can. J. Physiol. Pharmacol., 73 (1995) 1502-1511.

[20] A. Michel, R. Kaur, I. Chessell, P. Humphrey, Antagonist effects on human P2X7 receptor-mediated cellular accumulation of YO-PRO-1, Br. J. Pharmacol., 130 (2000) 513-520.

[21] C. Virginio, A. MacKenzie, F. Rassendren, R. North, A. Surprenant, Pore dilation of neuronal P2X receptor channels, Nat. Neurosci., 2 (1999) 315-321.

[22] F.B. Chekeni, M.R. Elliott, J.K. Sandilos, S.F. Walk, J.M. Kinchen, E.R. Lazarowski, A.J. Armstrong, S. Penuela, D.W. Laird, G.S. Salvesen, Pannexin 1 channels mediate/find-me/'signal release and membrane permeability during apoptosis, Nature, 467 (2010) 863-867.

[23] Y. Qu, S. Misaghi, K. Newton, L.L. Gilmour, S. Louie, J.E. Cupp, G.R. Dubyak, D. Hackos, V.M. Dixit, Pannexin-1 is required for ATP release during apoptosis but not for inflammasome activation, J. Immunol., 186 (2011) 6553-6561.

[24] C.C. Roth, R.A. Barnes Jr, B.L. Ibey, H.T. Beier, L.C. Mimun, S.M. Maswadi, M. Shadaram, R.D. Glickman, Characterization of Pressure Transients Generated by Nanosecond Electrical Pulse (nsEP) Exposure, Sci. Rep., 5 (2015).

[25] Z. Steelman, Z. Meng, A.J. Traverso, V.V. Yakovlev, Brillouin spectroscopy as a new method of screening for increased CSF total protein during bacterial meningitis, J. Biophotonics, 8 (2015) 408-414.

[26] A.J. Traverso, J.V. Thompson, Z.A. Steelman, Z. Meng, M.O. Scully, V.V. Yakovlev, Dual Raman-Brillouin microscope for chemical and mechanical characterization and imaging, Anal. Chem., 87 (2015) 7519-7523.

[27] O.V. Yarishkin, E.-M. Hwang, J.-Y. Park, D. Kang, J. Han, S.-G. Hong, Endogenous TRPM4-like channel in Chinese hamster ovary (CHO) cells, Biochem. Biophys. Res. Commun., 369 (2008) 712-717. 
[28] R. Skryma, N. Prevarskaya, P. Vacher, B. Dufy, Voltage-dependent Ca2+ channels in Chinese hamster ovary (CHO) cells, FEBS Lett., 349 (1994) 289-294. 